\title{
Density-dependent fecundity and infective juvenile production in the entomopathogenic nematode, Heterorhabditis megidis
}

\author{
J. J. RYDER* and C. T. GRIFFIN \\ Institute for Bioengineering and Agroecology, Department of Biology, National University of Ireland, Maynooth, \\ Co. Kildare, Ireland
}

(Received 24 December 2001 ; revised 4 March 2002; accepted 5 March 2002)

\begin{abstract}
S UMMARY
The entomopathogenic nematode Heterorhabditis megidis may undergo several rounds of reproduction within a single host. Infective juveniles (IJs) are formed within each generation during a process referred to as endotokia matricida, which involves the progressive consumption of the parent hermaphrodite or female by the developing IJs prior to emergence from the host cadaver. The present study examines the extent to which within-host population dynamics exhibit densitydependent variation. Particular attention is paid to the effect of infection density on the relative production of IJs and 'normal', non-infective offspring within each generation and on the emergence of the IJs from the host. Fecundity was found to be negatively density dependent across generations. However, at high infection density the first generation hermaphrodites invested relatively more in IJs at the expense of producing non-infective offspring. It is suggested that this pattern resulted from an adaptive, phenotypically plastic allocation of reproductive investment between offspring types in response to increased competition. The $F_{1}$ and $F_{2} \mathrm{IJ}$ s were also shown to emerge from the host in relatively discrete pulses.
\end{abstract}

Key words: Heterorhabditis, hermaphrodite, infective juvenile, density dependence.

\section{INTRODUCTION}

Entomopathogenic nematodes of the genus Heterorhabditis are lethal parasites of insects. Transmission occurs when specialized, developmentally arrested 3rd-stage juveniles (infective juveniles, or 'IJs') disperse from an expended host in order to locate and infect a new one (Poinar, 1990). There is no intermediate host and a variety of insect taxa can be parasitized. The IJs harbour symbiotic insectpathogenic bacteria (Photorhabdus spp.) within their intestine. Once a suitable host has been penetrated, the bacteria are released into the host's body cavity where they disable the immune system and cause the death of the host by septicaemia, usually within $48 \mathrm{~h}$ (Jarosz, 1998). This process creates a favourable environment for nematode growth and reproduction and enables the IJs to continue development through to adulthood.

The first $\left(P_{0}\right)$ generation of adults that develop within the host are hermaphroditic and produce offspring by one of two routes (Poinar, 1990). Eggs are initially laid into the host's body cavity where

* Corresponding author: School of Biological Sciences, Queen Mary and Westfield College, Mile End Road, London E1 4NS, UK. Tel: + 353 (0)1 6285222 Ext. 3136. Fax: +353 (0)1 708 3845. E-mail: jonryder_ie@ yahoo.com they undergo 'normal' development and give rise to a second $\left(F_{1}\right)$ generation of largely amphimictic adults (some hermaphrodites may also be present; Johnigk \& Ehlers, 1999a). However, a certain proportion of the eggs produced by the hermaphrodite are retained and hatch within the uterus. These offspring eventually develop into IJs during a process referred to as endotokia matricida (see Johnigk \& Ehlers, 1999b). The juveniles feed by consuming the parent's body tissues. Having disrupted the gonad, the 1 st- and 2nd-stage juveniles consume the intestine and have caused the death of the parent by the time they develop into IJs. Johnigk \& Ehlers $(1999 b)$ suggested that low food availability within the uterus may initiate determination of the 1st-stage juveniles into IJs. For this reason, a proportion of those eggs that were released into the host's body cavity may also develop into IJs when food availability is low (see also Fodor, Vecseri \& Farkas, 1990). Endotokia matricida ends with the migration of the IJs from the disintegrating parent's cuticle prior to emergence from the host cadaver (with the latter typically occurring between 1 and 2 weeks after infection in heterorhabditids).

Several rounds of reproduction may occur within a single host, with the $F_{1}$ females producing an $F_{2}$ generation of adults and IJs (the latter also developing by endotokia matricida) and the $F_{2}$ females producing further offspring themselves (Wang \& 
Bedding, 1996). Tens or hundreds of thousands of IJs may thus emerge from a single host cadaver. Clearly, however, the number and productivity of each generation of nematodes is likely to be constrained by the availability of resources within the host. As the number of IJs infecting a single host increases, for example, the level of competition for resources will also increase. Moreover, there will inevitably be high competition within the host between offspring in subsequent generations as nutritional conditions deteriorate.

Various life-history traits have been shown to exhibit density-dependent variation in entomopathogenic nematodes as a result of such competitionincluding survival, body size, fecundity, and time to and rate of IJ emergence (Zervos, Johnson \& Webster, 1991; Selvan, Campbell \& Gaugler, 1993; Koppenhöfer \& Kaya, 1995; Boff et al. 2000). However, although it is well known that several generations may occur within a single host, this factor has received little attention. For example, a common approach towards investigating densitydependent variation in fecundity is to pool together $\mathrm{IJ}$ s that emerge over a set period in order to compare yields between different infection treatments. Explicit consideration has not yet been given to whether those IJs are the product of a single generation or of multiple generations. Wang \& Bedding (1996) carried out a detailed investigation of within-host population dynamics in H. bacteriophora and Steinernema carpocapsae, but did so using a single infection density (Steinernema is a separate genus of entomopathogenic nematode with a very similar life-cycle). It remains unclear, therefore, how infection density affects the development of different generations within the host and how this corresponds to the pattern of IJ emergence. For example, does infection density affect the relative production of $\mathrm{IJ} s$ and 'normal', non-infective offspring within each generation and the emergence of the IJs? In an evolutionary sense, adult hermaphrodites or females are faced with a 'choice' when producing offspring within the host. At some point they must cease egg laying and allow the remainder of their offspring to undergo intra-uterine development and determination into the IJ stage (endotokia matricida): selection should clearly favour plasticity in this regard, if individuals that produce more second (or third) generation adult offspring than can be sustained by their host suffer reduced fitness.

The first aim of the present study, therefore, was to elucidate the extent to which within-host population dynamics exhibit density-dependent variation, by examining the relationship between infection density and the relative production of adult and IJ offspring by each generation within the host. The second aim was to correlate IJ production across generations with the pattern of emergence from the host at each infection density.
MATERIALS AND METHODS

\section{Insect and nematode cultures}

H. megidis (UK 211 strain) were cultured in vivo using wax moth larvae (Galleria mellonella) as hosts at $20^{\circ} \mathrm{C}$ in the dark. G. mellonella larvae were obtained from the Sheffield Mealworm Company (Sheffield, UK) and maintained at $15{ }^{\circ} \mathrm{C}$.

\section{Infection of hosts}

Replicate batches of $G$. mellonella larvae were exposed to 1 of 3 concentrations of IJs: 50, 500 or $1000 \mathrm{IJ}$ s per larva. IJ suspensions of the appropriate concentration were prepared by adjusting the concentration of pooled stock cultures (stored at $1000 \mathrm{IJs} / \mathrm{ml}$ ) by sedimentation. The concentration of a suspension was estimated from the mean number of IJs present in each of 5 samples taken after thorough mixing of the entire suspension. Infections were performed by adding $1 \mathrm{ml}$ of a suspension of the desired concentration to plastic food dishes $(9 \mathrm{~cm}$ diameter with snap-on lids; Roundstone Catering, Melksham, UK) lined with filter paper. G. mellonella larvae were then introduced into each dish and incubated at $20^{\circ} \mathrm{C}$ for 3 days. Ten replicate batches of larvae were exposed to each concentration of IJs, with each replicate (i.e. dish) containing 9 larvae selected at random from stock cultures. Three days after exposure, 7 infected larvae were selected at random, transferred to covered White traps (Woodring \& Kaya, 1988; each trap contained $50 \mathrm{ml}$ of tap water) and incubated at $20^{\circ} \mathrm{C}$ (the remaining 2 larvae were discarded).

\section{Within-host development and offspring production}

To investigate the effects of infection density on within-host development and offspring production, samples of larvae were dissected at intervals throughout the course of the infection. Four of the 10 replicate batches of infected larvae from each infection treatment $(50,500$ and $1000 \mathrm{IJ}$ s per host) were set aside for this purpose; the remaining 6 replicates were used for $\mathrm{IJ}$ harvesting in order to estimate emergence rates and total yield (see 'IJ emergence and harvesting' below). All replicates were otherwise maintained in exactly the same way, on White traps, throughout the infection. Preliminary studies had already indicated a suitable schedule for dissection, given that one of our intentions was to track the development and subsequent disintegration of the $P_{0}$ hermaphrodites and the $F_{1}$ and $F_{2}$ females through endotokia matricida. Accordingly, larvae were removed from the 4 'dissection' White traps on days 7, 12, 16, 20, 24 and 29 post-exposure; 5 larvae were removed for dissection on each day for each infection treatment (1 larva each from 3 of the traps and 2 from the fourth). Immediately upon removal, 
the larvae were placed in labelled tubes and frozen at $-20{ }^{\circ} \mathrm{C}$ until they could be thawed and dissected. IJ offspring were collected from the traps using the method described below (see 'IJ emergence and harvesting') and discarded.

Although freezing inevitably degrades some aspects of nematode morphology, it did not do so sufficiently to interfere with the identification of the various developmental stages (e.g. $P_{0}$ hermaphrodites, $F_{1} / F_{2}$ adults, IJs, etc). A proportion of the $F_{1}$ and $F_{2}$ adults that were designated as 'female' were probably hermaphrodites (Johnigk \& Ehlers, 1999a). However, because these individuals could not be identified as such unequivocally at the times on which dissections were performed, they were also categorized as 'female' and will be referred to as such below unless otherwise indicated.

Several parameters were recorded for each infection treatment. First, the total numbers of adult hermaphrodites and/or females and males from each generation were determined on days $7,12,16,20,24$ and 29 post-exposure. Dissections were performed by removing the host larva's cuticle under a binocular microscope (Leica, Switzerland) and gently separating the remaining aggregate of body tissues and nematodes in tap water using a pipette. Adults can clearly be distinguished from juveniles by the presence of a fully formed gonad. As one of our main aims was to examine the effects of infection density on fecundity and IJ production, only adult and IJ offspring were included in the subsequent analysis. 'Normal' (non-infective) juveniles were not counted. Adults from the first 3 generations can largely be distinguished on the basis of size alone, but also developed in relatively discrete temporal pulses under the conditions used in this study (see Results section). In order to track the disintegration of adult hermaphrodites or females by endotokia matricida, only fully intact adults were counted at each time-interval. If there were any signs that the cuticle had begun to rupture, allowing the exit of IJs into the host's haemocoel, those adults were not counted.

Second, the mean number of adult hermaphrodites present in the hosts on days 7 and 12 combined ( $n=10 \mathrm{G}$. mellonella larvae per infection treatment) was taken as an estimate of mean infection density (i.e. the mean number of $\mathrm{IJ}$ s that successfully established in the host larvae and completed development through to the adult hermaphrodite stage) for each treatment. Disintegration did not begin to take place until sometime after 12 days post-exposure (see Results section). In addition, preliminary studies had indicated that very little mortality occurs in the $P_{0}$ generation prior to the onset of endotokia matricida at the infection concentrations chosen for this study.

Third, the numbers of IJ and adult offspring produced per parent hermaphrodite or female were determined for each infection density for the $P_{0}$ and $F_{1}$ generations. The number of adult offspring was calculated as the number of $F_{1}$ or $F_{2}$ adults, respectively, per parent hermaphrodite or female, using the mean number of adult $P_{0}$ hermaphrodites or $F_{1}$ females. In order to estimate the number of IJ offspring that were produced, a maximum of 4 adult $P_{0}$ hermaphrodites and $F_{1}$ females were dissected per host larva $(n=5)$ per time-interval per infection treatment. Only fully intact adults were dissected, and were taken as near as possible, but prior to, the point at which IJs began to emerge from their parent's cuticle. The hermaphrodites or females were dissected individually under a dissecting microscope and the number of IJs was counted. In a small number of cases on the days chosen, hermaphrodites or females contained very low numbers of 1 st- or 2nd-stage juveniles, in addition to $\mathrm{IJs}$, and these were not counted. Offspring production could not be analysed for the $F_{2}$ generation due to logistic considerations. However, very few adult offspring were produced per $F_{1}$ female (see Results section), which greatly restricted the potential production of $F_{3}$ offspring. The $F_{2}$ females were also found to each produce fewer IJs than the $F_{1}$ females, further reducing the potential yield of $F_{3}$ IJs.

\section{If emergence and harvesting}

Each of the 6 White traps set aside for IJ harvesting were checked daily for the presence of IJs from day 12 until day 29 post-exposure (by which time IJ yields had become very low). When IJs were found to be present, the suspension was transferred to a measuring cylinder along with another $50 \mathrm{ml}$ of tap water used to rinse any remaining IJs from the trap. The number of IJs in the harvest was then estimated from the mean number present in each of 5 samples taken after thorough mixing. Daily IJ emergence rates and total yields were used to estimate the rate of IJ production per host per $P_{0}$ generation hermaphrodite. The harvested IJs also formed the subject of a separate study on infectivity (Ryder \& Griffin, unpublished observations).

\section{Statistical analysis}

The effect of infection density on each of the experimental parameters under consideration was examined using Analysis of Variance (ANOVA; Minitab Statistical Software, release 13.1 for the PC). Data were checked for normality and homogeneity of variances and transformed where appropriate. Where possible, a factorial design was used, as is indicated in the text. However, in most cases variances were too heterogeneous between experimental factors (e.g. offspring type) to allow for a single transformation (Underwood, 1998). Separate one-way ANOVAs were then carried out for each individual factor. 
RESULTS

\section{Infection densities}

There was a significant increase in infection density (i.e. the mean number of adult $P_{0}$ hermaphrodites present in the hosts) when hosts were exposed to higher concentrations of IJs (log-transformed data: $\mathrm{F}=36 \cdot 75$, D.F. $=2,27, P<0 \cdot 001)$, with infection density differing significantly between each of the 3 concentrations ('Table 1). However, there was no significant effect of IJ concentration on the mean proportion that successfully established in the hosts (log-transformed data: $\mathrm{F}=0 \cdot 48$, D.F. $=2,27, P=$ $0 \cdot 626)$, suggesting that infection rates were approximately constant (Table 1).

\section{Relative production of adult and IF offspring within the host}

The production of adult female and IJ offspring was determined across infection densities (i.e. 16, 103 and $424 P_{0}$ hermaphrodites per host; see Table 1) for the $P_{0}$ hermaphrodites and the $F_{1}$ females. Because male $F_{1}$ offspring do not contribute directly to IJ production, data for males are presented separately (see below). There was a significant reduction in the mean number of adult females produced per $P_{0}$ hermaphrodite as infection density increased (log-transformed data: $\mathrm{F}=29 \cdot 25, \quad$ D.F. $=2,12$, $P<0 \cdot 001)$. For example, on average the $P_{0}$ hermaphrodites produced $190.6 F_{1}$ females each at the lowest infection density $\left(16 P_{0}\right.$ hermaphrodites per host) compared with only 15.9 at the highest infection density ( $424 P_{0}$ hermaphrodites per host; see Fig. 1A for pair-wise comparisons). There was also a significant reduction in the mean number of IJs produced per $P_{0}$ hermaphrodite with increasing infection density $(\mathrm{F}=7 \cdot 16$, D.F. $=2,12, P<0 \cdot 01)$. On average, the $P_{0}$ hermaphrodites produced $577 \cdot 7 F_{1}$ IJs each at the lowest infection density compared with only 248.7 at the highest infection density (Fig. 1A).

In addition, there were significant differences in both the mean number of adult females and the mean number of IJs produced per $F_{1}$ female as infection density increased (two-way ANOVA, log-transformed data: offspring type: $\mathrm{F}=1484.95$, D.F. $=$ 1,24, $P<0.001$; infection density: $\mathrm{F}=72 \cdot 15$, D.F. $=$ 2,24, $P<0 \cdot 001)$. The $F_{1}$ females produced very few $F_{2}$ females $(<1$ each) at any infection density but produced greater numbers of IJs, with the average number declining from $77 \cdot 5$ at the lowest infection density to $9 \cdot 6$ at the highest infection density (see Fig. 1B for pair-wise comparisons). Consequently, there was a significant interaction between the effects of offspring type and infection density on the mean number of offspring produced (log-transformed data: $\mathrm{F}=12 \cdot 63$, D.F. $=2,24, P<0 \cdot 001)$.

Fig. 2 shows the proportion of offspring that developed into IJs in the $F_{1}$ and $F_{2}$ generations across the 3 infection densities, expressed as a proportion of the combined number of adult female and IJ offspring within each generation. There was a significant increase in the proportion of offspring that developed into $\mathrm{IJ} s$ in the $F_{1}$ generation as infection density increased (arcsine-transformed data: $\mathrm{F}=13.67$, D.F. $=2,12, P<0.01)$. In contrast, there was a significant decrease in the proportion that developed into IJs in the $F_{2}$ generation (arcsinetransformed data: $\mathrm{F}=11 \cdot 71$, D.F. $=2,12, P<0 \cdot 01$ ), although it may be noted that very few $F_{2}$ females were produced by the $F_{1}$ females at any infection density (see above).

Adult males comprised a small proportion of the total number of adult $F_{1}$ offspring at each infection density (mean proportion \pm s.E.: $0 \cdot 13 \pm 0 \cdot 007$ at $16 P_{0}$ hermaphrodites per host; $0 \cdot 15 \pm 0 \cdot 007$ at 103 $P_{0}$ hermaphrodites per host; $0 \cdot 28 \pm 0.033$ at $424 P_{0}$ hermaphrodites per host). However, there was a significant increase in the proportion that were male as infection density increased (Kruskal-Wallis test: $\mathrm{H}=9 \cdot 07$, D.F. $=2, P=0 \cdot 01)$. Very few male $F_{2}$ adults were observed at any infection density on the days on which dissections were performed (typically $<10$ per host cadaver).

\section{Disintegration of $\mathrm{P}_{0}$ hermaphrodites and $\mathrm{F}_{1}$ females} by endotokia matricida

Fig. 3 shows the development and subsequent disintegration within the host of adult hermaphrodites and females by endotokia matricida, with time after exposure. There was a significant effect of time on the mean number of intact adult hermaphrodites or females present in each generation, at each of the 3 infection densities $(P<0.001$; Table 2$)$. The arrows on each figure denote the periods during which adult hermaphrodites ( $P_{0}$ generation) or females ( $F_{1}$ generation) were found to have disintegrated, as indicated by a significant drop in numbers between adjacent time-points (Tukey pair-wise comparisons, $P<0.05$ ). (Those $F_{2}$ females that were present had not begun to disintegrate by the time dissections ceased.)

At the lowest infection density (16 $P_{0}$ hermaphrodites per host), the number of intact $P_{0}$ hermaphrodites dropped significantly from day 16 to day 20 (reaching zero on day 20), suggesting that disintegration began at or around day 16 (see Fig. 3A). The number of intact $F_{1}$ females dropped significantly from day 20 to day 24 and dropped to zero by day 29 , suggesting that disintegration began at some point after day 20. On days 16 and 20, a number of $F_{1}$ females were observed to lack the morphological characteristics indicative of endotokia matricida. These females had an opaque appearance suggestive of microbial decay and comprised an average of $12.0 \% \quad( \pm 4 \%$ s.E. $)$ and $9.9 \% \quad( \pm 6 \%$ s.E. $)$, respectively, of the total number of $F_{1}$ females. 
Table 1. Mean infection parameters ( \pm s.E.) following exposure to different concentrations of IJs

\begin{tabular}{|c|c|c|c|}
\hline & \multicolumn{3}{|c|}{ Number IJs/host } \\
\hline & 50 & 500 & 1000 \\
\hline Infection density* & $15 \cdot 7 a \pm 5 \cdot 2$ & $102 \cdot 8 b \pm 18 \cdot 3$ & $423 \cdot 6 c \pm 138 \cdot 3$ \\
\hline Proportion of IJs & $0 \cdot 31 \pm 0 \cdot 10$ & $0 \cdot 21 \pm 0 \cdot 04$ & $0 \cdot 42 \pm 0 \cdot 14$ \\
\hline
\end{tabular}

* Means with different letter codes differ significantly at $P<0 \cdot 05$ (Tukey pairwise comparisons).

A
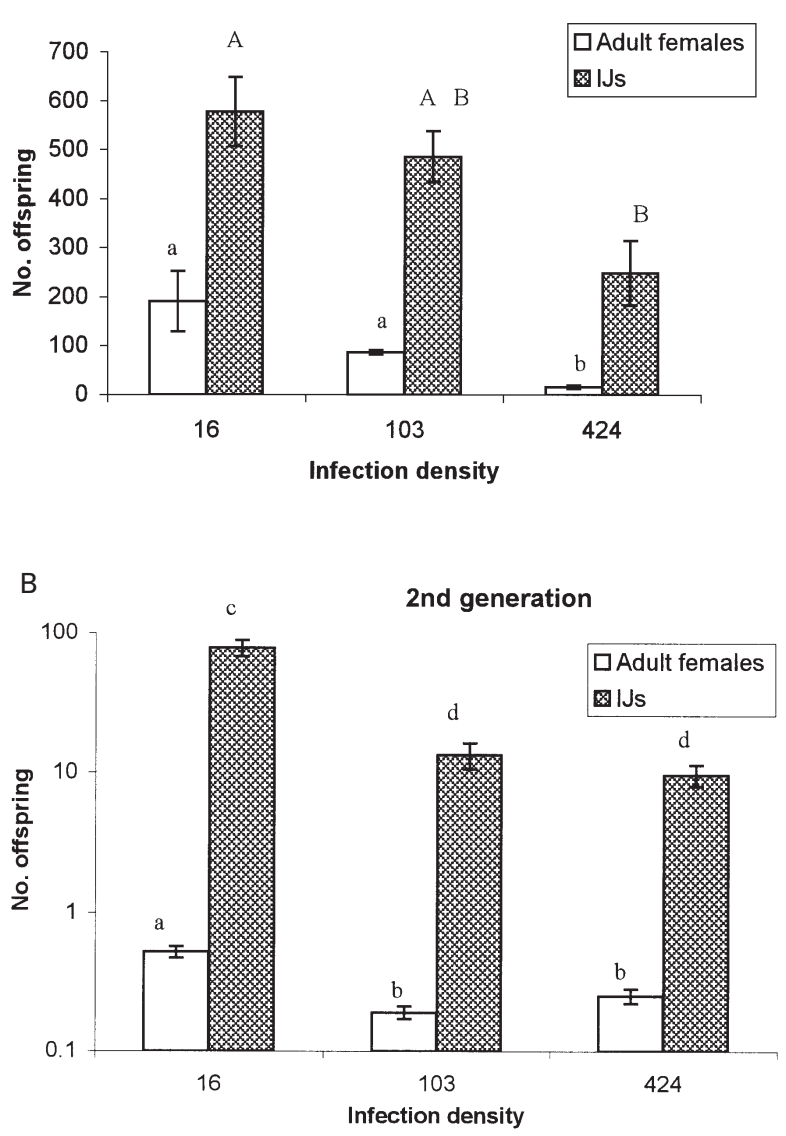

Fig. 1. Number of adult female and IJ offspring produced at each infection density (A) per $P_{0}$ hermaphrodite and (B) per $F_{1}$ female. Means sharing same letter codes do not differ significantly at $P<0 \cdot 05$ (Tukey pair-wise comparisons. Different letter codes are used for adult female and IJ offspring on Fig. 1A because it was necessary to analyse the data by separate one-way ANOVAs; a single letter code is used for Fig. $1 \mathrm{~B}$ as the data were suitable for a two-way ANOVA).

Approximately 5 of these females were dissected for each host larva (with $n=5$ larvae on each day) and on no occasion did these contain infective juvenile offspring; instead, all contained large amounts of dark brown amorphous material and occasionally small numbers of 1 st- or 2nd-stage juveniles. Overcrowding presumably therefore led to the death of a proportion of the $F_{1}$ females, such that their decay partially accounts for the drop in the number of $F_{1}$ females between days 20 and 24 (with disintegration through endotokia matricida accounting for the remainder).

At the intermediate infection density (103 $P_{0}$ hermaphrodites per host), the number of intact $P_{0}$ hermaphrodites dropped significantly from day 12 to day 16 and reached zero by day 20 , suggesting that disintegration began at some point after day 12 (see Fig. 3B). The number of intact $F_{1}$ females dropped significantly from day 16 to day 20 and reached zero by day 24 , suggesting they began to disintegrate at some point around day 16 . However, a substantial number of $F_{1}$ females were observed on day 16 which had the 'opaque' appearance and morphology suggestive of decomposition $(22.6 \% \pm 6 \cdot 6$ s.E. of the total number of $F_{1}$ females on that day). These females had apparently decayed completely soon afterwards, as none was present by day 20 or thereafter. Thus, over-crowding probably also lead to the decomposition of a proportion of the $F_{1}$ females at the intermediate infection density.

At the highest infection density (424 $P_{0}$ hermaphrodites per host), essentially the same pattern was recorded as that found for the intermediate infection density (see Fig. 3C). Significant reductions in the numbers of $P_{0}$ hermaphrodites and $F_{1}$ females occurred after days 12 and 16, respectively. A number of 'opaque' $F_{1}$ females were also recorded on day $16\left(12.0 \% \pm 4\right.$ s.E. of the total number of $F_{1}$ females) which had decomposed completely by day 20 .

\section{Daily IF emergence rates}

At each infection density, IJs were found to emerge from the host cadavers in 2 distinct pulses (Fig. 4). At the lowest infection density $\left(16 P_{0}\right.$ hermaphrodites per host), the first pulse reached a maximum on day 20; the second pulse reached a maximum on day 25. Each pulse must therefore largely reflect the emergence of the $F_{1}$ and $F_{2}$ IJs, respectively. Maximum IJ emergence in the first pulse occurred within 4 days of the onset of disintegration of the $P_{0}$ hermaphrodites and prior to the disintegration of the $F_{1}$ females. Maximum IJ emergence in the second pulse occurred within 5 days of the onset of 


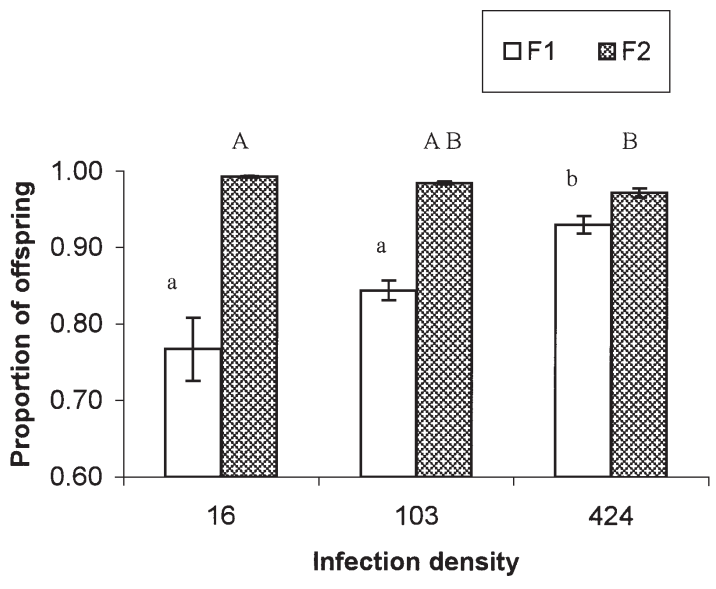

Fig. 2. Proportion of (non-male) $F_{1}$ and $F_{2}$ offspring that developed into IJs at each infection density. For each generation, means sharing same letter codes do not differ significantly at $P<0.05$ (Tukey pair-wise comparisons following one-way ANOVA).

disintegration of the $F_{1}$ females and 5 days after the maximum rate of emergence for the $F_{1}$ IJs (see Fig. $3 \mathrm{~A})$. At the intermediate $\left(103 P_{0}\right.$ hermaphrodites per host) and highest (424 $P_{0}$ hermaphrodites per host) infection densities, the maximum rates of emergence for the first and second pulse occurred on day 19 and day 23, respectively. Again, these pulses must largely reflect the emergence of $F_{1}$ and $F_{2}$ IJs, respectively. Maximum IJ emergence in the first pulse occurred within 7 days of the onset of disintegration of the $P_{0}$ hermaphrodites and probably no more than 3 days after the onset of disintegration of the $F_{1}$ females (see Fig. $3 \mathrm{~B}$ and $\mathrm{C}$ ). Maximum IJ emergence in the second pulse occurred within 7 days of the onset of disintegration of the $F_{1}$ females and 4 days after the maximum rate of emergence for the first pulse.

A certain amount of cross-contamination of one emergence pulse with IJs from the other pulse may have occurred at each infection density. At the lowest infection density, the first pulse must have been comprised almost entirely of $F_{1}$ IJs (see above). Small numbers of $F_{1}$ IJs probably emerged with the $F_{2}$ IJs in the second pulse, but these would have comprised a very small fraction of the total number that emerged (given the 100-fold rise in IJ yield). At the higher infection densities (103 and 424 IJs per host) more cross-contamination would have been possible. However, the decomposition of a substantial proportion of the $F_{1}$ females around day 16 suggests that the onset of endotokia matricida may be exaggerated in Fig. $3 \mathrm{~B}$ and $\mathrm{C}$ for the $F_{1}$ females. Few $F_{2}$ IJs may therefore have been in a position to emerge from the host until closer to 20 days postexposure -1 day or more after the maximum rate of IJ emergence in the first pulse. Moreover, daily IJ emergence rates dropped substantially at each infection density towards the 'tail' of the first pulse, prior to the onset of the second pulse (see Fig. 4).

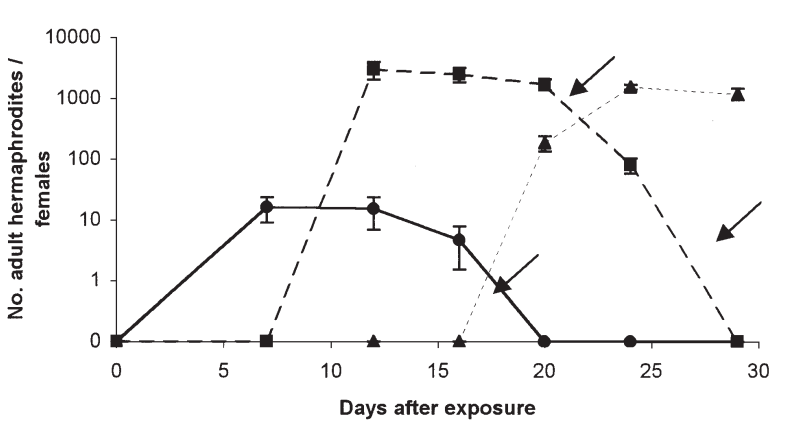

B

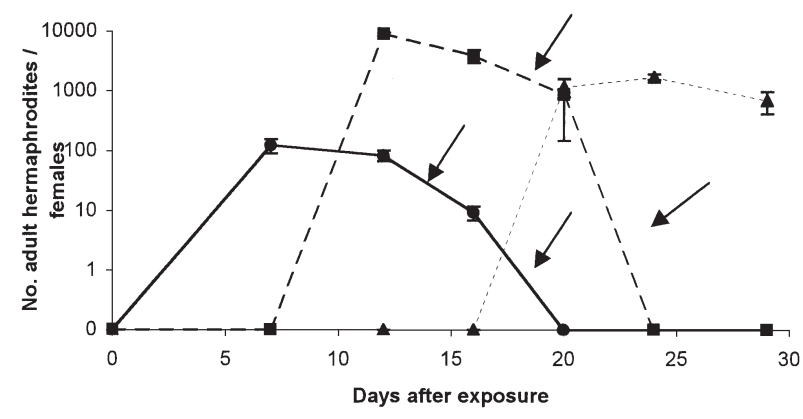

C

424 hermaphrodites per host

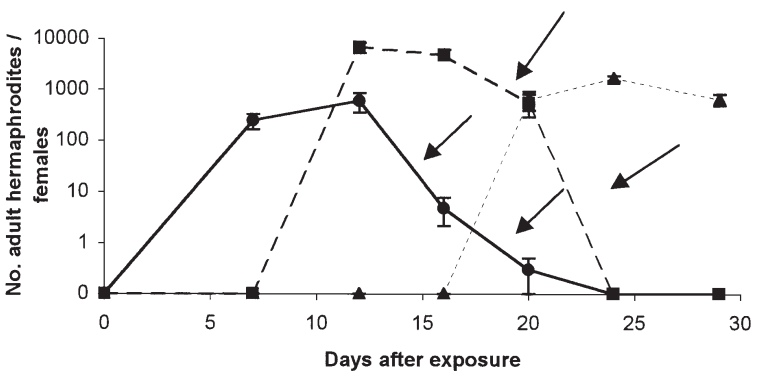

Fig. 3. Mean number of intact adult hermaphrodites and females present in hosts at each infection density with time after exposure. (A), (B) and (C) show numbers for 16, 103 and $424 P_{0}$ hermaphrodites per host, respectively. (- - - ) $P_{0}$ hermaphrodites; (-- $\left.\mathbf{- -}\right) F_{1}$ females; (-- $\mathbf{\Delta}--) F_{2}$ females. Only fully intact adults are shown; arrows indicate where significant drops occurred between time-intervals (Tukey pair-wise comparisons, $P<0 \cdot 05$. Note: Tukey tests were carried out for comparisons between all time-intervals within generations, but only those corresponding to adult disintegration are shown on the figure for clarity; see text for details).

\section{Components of IF yield}

IJ yield, expressed per host per $P_{0}$ hermaphrodite, was negatively density dependent. Yields were compared across infection densities for the first and second emergence pulses and for all days combined. The sums of the daily IJ yields within each pulse provide estimates of the yield of $F_{1}$ and $F_{2}$ IJs that emerged from the host. The second pulse was considered to have begun by day 23 for the lowest 
Table 2. One-way analyses of variance (F-ratios) for the effect of time after exposure on the number of intact adult hermaphrodites or females at each infection density

\begin{tabular}{lccr}
\hline \hline & \multicolumn{3}{l}{ Infection density } \\
\cline { 2 - 4 } & 16 & 103 & \multicolumn{1}{c}{424} \\
\hline$P_{0}$ hermaphrodites & $20 \cdot 69$ & $239 \cdot 38$ & $69 \cdot 56$ \\
$F_{1}$ females & $72 \cdot 94$ & $50 \cdot 57 *$ & $254 \cdot 93$ \\
$F_{2}$ females & $62 \cdot 30$ & $67 \cdot 13$ & $62 \cdot 30$ \\
\hline \hline
\end{tabular}

* F-ratio for non-transformed data, all other ratios derived from $\log$-transformed data: D.F. $=6,28, P<0 \cdot 001$.

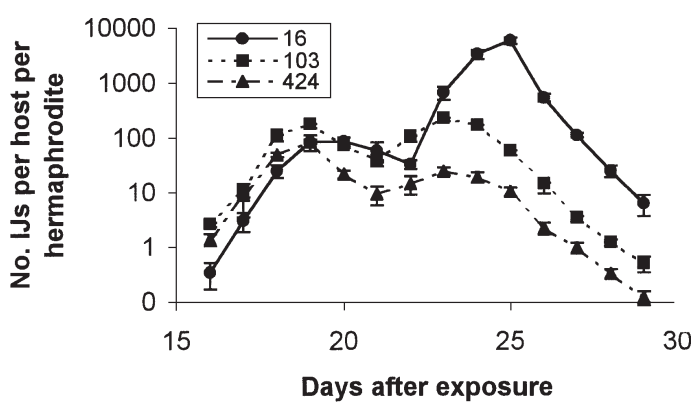

Fig. 4. Mean daily IJ yield per host cadaver per $P_{0}$ hermaphrodite for each infection density.

infection density and day 22 for the intermediate and highest infection densities, judging from the availability of IJs from parental disintegration (see Fig. 3) and the observed increases in mean IJ yield (see Fig. 4). There was a significant effect of infection density on mean IJ yield for the first emergence pulse (log-transformed data: $\mathrm{F}=15 \cdot 16$, D.F. $=2,15, P<$ $0 \cdot 001)$. Mean yield was slightly but significantly higher for the intermediate infection density than for the lowest infection density, and slightly but significantly higher for the lowest than for the highest infection density (see Table 3). However, there was a consistent and significant decrease in mean IJ yield for the 2 nd emergence pulse as infection density increased (log-transformed data: $\mathrm{F}=382 \cdot 36$, D.F. $=$ $2,15, P<0 \cdot 001)$. Mean yield per $P_{0}$ hermaphrodite dropped from 10544 at the lowest infection density to only $72 \cdot 8$ at the highest infection density (Table 3 ). Consequently, there was a significant and substantial reduction in mean total yield per $P_{0}$ hermaphrodite as infection density increased (logtransformed data: $\mathrm{F}=1252 \cdot 72, \quad$ D.F. $=2,15, P<$ $0 \cdot 001)$, dropping from an average of $10833.8 \mathrm{IJs}$ at the lowest infection density to only $247 \cdot 4$ at the highest infection density (see Table 3 ). Total yield per host was also negatively density dependent between the lowest and the intermediate and the lowest and highest infection densities $(\mathrm{F}=34.97$, D.F. $=2,15$, $P<0 \cdot 001)$, even though per host yields do not control for variation in the number of parent hermaphrodites that were present (Table 3).

Table 3 also compares the total numbers of IJs that emerged in the first and second pulses with estimates of the total numbers of $F_{1}$ and $F_{2}$ IJs produced within the host per $P_{0}$ hermaphrodite, as determined by dissection (see 'Relative production of adult and IJ offspring within the host' above). Although only about half of the individual comparisons revealed significant differences (possibly due to small sample sizes: $n=6$ for the emergence yields and $n=5$ for dissection data), the overall pattern is highly consistent and suggests that more IJs tended to be produced per $P_{0}$ hermaphrodite than actually emerged (see Table 3; Mann-Whitney tests for emergence yields and dissection data for each infection density): first pulse $/ F_{1}$ IJs : $16 P_{0}$ hermaphrodites per host: $\mathrm{W}=23 \cdot 0, \mathrm{P}=0.023 ; 103 P_{0}$ hermaphrodites per host: $\mathrm{W}=31 \cdot 0, \mathrm{P}=0 \cdot 411 ; 424$ $P_{0}$ hermaphrodites per host: $\mathrm{W}=30 \cdot 0, \mathrm{P}=0 \cdot 315$; second pulse $/ F_{2}$ IJs: $16: \mathrm{W}=25 \cdot 0, \mathrm{P}=0.055 ; 103$ : $\mathrm{W}=21 \cdot 0, \mathrm{P}=0 \cdot 008 ; 424: \mathrm{W}=23 \cdot 0, \mathrm{P}=0 \cdot 023$; first and second emergence pulses $\left(F_{1}\right.$ and $F_{2}$ IJs) combined : $16: \mathrm{W}=25 \cdot 0, \mathrm{P}=0 \cdot 055 ; 103: \mathrm{W}=21 \cdot 0$, $\mathrm{P}=0.008 ; 424: \mathrm{W}=29 \cdot 0, \mathrm{P}=0 \cdot 235$. The statistical analysis for the number of $F_{1}$ IJs produced per $P_{0}$ hermaphrodite across infection densities (based on dissection data) was presented earlier in this section and is not repeated on Table 3 . The analyses for the number of $F_{2}$ IJs dissected per $P_{0}$ hermaphrodite, and $F_{1}$ and $F_{2}$ IJs combined, exactly mirrored those based on the emergence data (see Table 3) and are therefore not presented.

\section{ISCUSSION}

Once a $H$. megidis IJ has successfully located and infected a new host, it resumes development and reaches sexual maturity in the adult hermaphrodite stage ( $P_{0}$ generation). It then produces offspring by 2 different routes. Initially, eggs are released into the host's body cavity and give rise to an $F_{1}$ generation of amphimictic adults (Johnigk \& Ehlers, 1999a), which eventually produce further $\left(F_{2}\right)$ adult and IJ offspring. In contrast, eggs that are retained within the uterus develop directly into $F_{1}$ IJs by endotokia matricida (Johnigk \& Ehlers, 1999b). The present study has demonstrated that total fecundity is density dependent in both the $P_{0}$ and $F_{1}$ generations. Thus, at high infection density the $P_{0}$ hermaphrodites produced fewer IJ and adult offspring and the $F_{1}$ adult females produced substantially fewer IJs. More interestingly, however, it was also found that a lower proportion of the offspring of the $P_{0}$ hermaphrodites developed into $F_{1}$ females at high infection density, with the majority instead developing into IJs. Because little mortality was observed in the $F_{1}$ generation prior to adulthood, this effect almost 
Table 3. Mean yield of IJs that emerged per host per $P_{0}$ hermaphrodite $( \pm$ s.E.) within the first, second, and first and second pulses combined at each infection density (see text for details)

(For comparison, data in italics show the mean numbers of $F_{1}$ and $F_{2}$ IJs produced within the host per $P_{0}$ hermaphrodite prior to emergence, as determined by dissection. Means with different letter codes differ significantly at $P<0 \cdot 05$ (lower case: Tukey pair-wise comparisons within rows for emergence data only; upper case: Mann-Whitney tests for within column comparisons of IJ numbers estimated from emergence yields and dissection data, for $F_{1}, F_{2}$, and $F_{1}$ and $F_{2}$ IJs combined). See text for definition of 'pulse'.)

\begin{tabular}{|c|c|c|c|}
\hline & \multicolumn{3}{|l|}{ Infection density } \\
\hline & 16 & 103 & 424 \\
\hline First pulse & $289 \cdot 8 \pm 44 \cdot 8 a \mathrm{~A}$ & $418.5 \pm 32.9 \mathrm{bA}$ & $174 \cdot 6 \pm 8 \cdot 5 c A$ \\
\hline$F_{1} I \mathcal{F}$ production & $577 \cdot 7 \pm 70 \cdot 6 B$ & $486 \cdot 3 \pm 52 \cdot 2 \mathrm{~A}$ & $248 \cdot 7 \pm 66 \cdot 1 \mathrm{~A}$ \\
\hline Second pulse & $10544 \cdot 0 \pm 469 \cdot 0 \mathrm{aA}$ & $591.5 \pm 41.5 \mathrm{bA}$ & $72 \cdot 8 \pm 11 \cdot 1 \mathrm{cA}$ \\
\hline$F_{2} I \mathcal{F}$ production & $14765 \cdot 0 \pm 1971 \cdot 0 \mathrm{~A}$ & $1164 \cdot 0 \pm 239 \cdot 0 \mathrm{~B}$ & $152 \cdot 8 \pm 28 \cdot 6 B$ \\
\hline Total & $10833.8 \pm 463.8 \mathrm{aA}$ & $1010 \cdot 0 \pm 51 \cdot 5 \mathrm{bA}$ & $247 \cdot 4 \pm 15 \cdot 4 c A$ \\
\hline$F_{1}+F_{2} I \mathcal{F}$ & $15343 \cdot 0 \pm 1919 A$ & $1650 \cdot 0 \pm 256 \cdot 0 \mathrm{~B}$ & $401 \cdot 5 \pm 82 \cdot 2 \mathrm{~A}$ \\
\hline Total (per host) & $170091 \cdot 0 \pm 7281 \cdot 7 a$ & $103824 \cdot 3 \pm 5297 \cdot 4 b$ & $104784 \cdot 6 \pm 6537 \cdot 2 b$ \\
\hline
\end{tabular}

certainly arose from a decrease in the number of eggs released into the host. Thus, although fecundity was negatively density dependent in the $P_{0}$ and $F_{1}$ generations, relative investment in $\mathrm{IJ} s$ by the $P_{0}$ hermaphrodites was positively density dependent.

Some of the eggs released into the host cadaver by the $P_{0}$ hermaphrodites may have developed into IJs at high infection density instead of undergoing 'normal' development. This is because food resources would have been depleted more rapidly at high infection density, potentially inducing juvenile offspring to develop into IJs within the cadaver as well as within the parent hermaphrodite (Johnigk \& Ehlers, $1999 b$ ). This effect would also have tended to increase the proportion of $F_{1}$ offspring that developed into IJs at high infection density. However, IJs were rarely detected in the host cadavers prior to the onset of endotokia matricida in the $P_{0}$ generation. At that point, sufficient time had elapsed to enable most non-infective $F_{1}$ offspring to reach adulthood, indicating that very few $F_{1}$ offspring are likely to have developed into IJs except by endotokia matricida.

One possible explanation for the above finding is that the $P_{0}$ hermaphrodites adaptively adjusted their reproductive investment between the two different types of offspring in relation to the level of competition. At high infection density the $P_{0}$ hermaphrodites appear to have increased investment in IJs at the expense of 'normal', non-infective juveniles. This strategy may have served to minimize the negative effects of competition on offspring development and survival. Infective juvenile offspring obtain the resources they require by consuming their mother. They are then capable of dispersing away from the original host cadaver in order to attempt infection of a new host. Noninfective offspring, however, must rely on the continued availability of resources within the original host in order to reach sexual maturity and produce $F_{2}$ offspring, and are therefore likely to have been more constrained at high infection density. At low infection density, the fitness of the $P_{0}$ hermaphrodites was reflected in relatively high $F_{1}$ IJ production-and, indirectly, in extremely high $F_{2}$ IJ production. However, because fecundity was negatively density dependent in both the $P_{0}$ and $F_{1}$ generations, at high infection density the $P_{0}$ hermaphrodites would not have had the option of achieving fitness gains indirectly to the same extent - i.e. via the reproductive success of their noninfective $F_{1}$ offspring. If the $P_{0}$ hermaphrodites had produced as many non-infective offspring at high infection density as at low density, those offspring would inevitably have suffered even lower fecundity. Data presented in the Results section also suggest that a proportion of the $F_{1}$ adult females may have died at each infection density due to overcrowding. Thus, had higher numbers of $F_{1}$ adult females been produced at higher infection densities, they may also have suffered higher mortality.

Our data only permit speculation regarding the precise fitness consequences of differentially apportioning reproductive investment between IJs and non-infective offspring. Nevertheless, it is tempting to suggest that a phenotypically plastic pattern of allocation is possible in response to increased competition (Roff, 1997). For example, at high infection density the $P_{0}$ hermaphrodites may suffer lower inclusive fitness if they release too many eggs into the host, if as a consequence those offspring suffer reduced fecundity or survival. However, this would depend on a number of parameters that were not quantified in the present study, which may in themselves be density dependent - such as the proportion of amphimictic and hermaphroditic individuals in the $F_{1}$ generation and the rate of self- and cross-fertilization. It should also be noted that these 
results do not provide direct evidence that the response of the $P_{0}$ hermaphrodites of allocating more reproductive investment into $F_{1} \mathrm{IJ}$ s at higher infection density was under behavioural control. Lower rates of egg laying may simply have been a consequence of some unknown constraint on egg production or release (interestingly, the $F_{1}$ females tend to be smaller at high infection density; J. Ryder, unpublished observations; see also Selvan et al. 1993).

Although we found that the $P_{0}$ hermaphrodites invested relatively more in $\mathrm{IJs}$ at high infection density, a greater proportion of those adult $F_{1}$ offspring that were produced developed as males. Previous studies on $H$. megidis (HSH1 strain; Strauch, Stoessel \& Ehlers, 1994) and H. bacteriophora (HP88 strain; Kahel-Raifer \& Glazer, 2000) suggest that a greater proportion of heterorhabditid $F_{1}$ offspring develop as hermaphrodites when nutritional conditions are poor, but neither study detected a change in the sex ratio of the amphimictic offspring. Because we did not distinguish between hermaphrodites and females in the $F_{1}$ generation, we cannot infer the precise amphimictic sex ratio (i.e. females:males). It is therefore possible that the increase we detected in the proportion of male offspring was an artefact arising from a change in the proportion of hermaphrodites relative to females amongst the adult $F_{1}$ offspring. However, this seems unlikely given the results of Strauch et al. (1994) and Kahel-Raifer \& Glazer (2000), because it would require that the proportion of individuals that developed as hermaphrodites decreased in response to nutritional stress. Interestingly, Dix et al. (1994) demonstrated that the sex ratio of the offspring of first generation Heterorhabditis hermaphrodites (HP 88 strain) increased from a negligible proportion of males to approximately $5 \%$ when the hermaphrodites used their own sperm to fertilize their eggs rather than the sperm of out-crossed males. However, in the present study the $P_{0}$ hermaphrodites would probably only have been able to use their own sperm to fertilize their eggs, as the $F_{1}$ males would probably have reached sexual maturity too late.

This study has demonstrated that $F_{1}$ and $F_{2} H$. megidis IJs emerge in discrete 'pulses' from the host. Although IJ production was shown to be negatively density dependent in the $F_{1}$ generation (based on the dissection of $P_{0}$ hermaphrodites), total emergence for the first pulse (the $F_{1} \mathrm{IJ}$ ) was highest at the intermediate infection density. Whether this effect resulted from behavioural differences associated with the conditions experienced by the $F_{1}$ IJs within the host cadaver, or from some other factor, is not known. For example, the $F_{1}$ IJs may have been more active and therefore faster to emerge from the host at the intermediate infection density. A comparison of the mean number of $\mathrm{IJ} s$ produced per $P_{0}$ hermaphrodite within the host with the mean number that emerged suggests that a substantial proportion of the IJs frequently failed to emerge within the period covered in this study. However, there was little indication that this effect was density dependent. It is also possible that it resulted from differential IJ mortality within the hosts.

Emergence rates were negatively density dependent for the $F_{2}$ IJs, with the total yield of IJs per host per $P_{0}$ hermaphrodite also showing strong negative density dependence. These findings agree well with previous studies that have examined the effect of infection density on IJ yield in entomopathogenic nematodes. For example, Zervos et al. (1991) showed that the yield of IJs from $G$. mellonella infected with $H$. heliothidis was negatively density dependent per inoculated IJ, under approximately equivalent experimental conditions. Because Zervos et al. (1991) determined yield for the entire period over which IJs emerged, it is likely that the differences recorded between infection densities mask some variation in the contribution of each generation that developed within the host. Boff et al. (2000) obtained very similar results for $H$. megidis (also using the UK 211 strain) per $P_{0} \mathrm{IJ}$, but only determined IJ yield for the first 5 days after emergence began.

The pattern of emergence from the host may be subject to strong selection in entomopathogenic nematodes. Stuart, Lewis \& Gaugler (1996) selected for early and late emergence using wild isolates of $S$. glaseri. Sufficient genetic variation was present to permit a significant increase in the time to first emergence in the 'slow' line after 12 rounds of selection, but there was no change in the line selected for 'fast' emergence relative to a control line. Stuart et al. (1996) suggested that early emergence may often be under particularly strong selection, for example when competition for nearby hosts is intense. Nevertheless, genetic variation may sometimes be maintained by temporal or spatial variation in the availability of susceptible hosts, or by tradeoffs with correlated traits such as those associated with resource use, IJ formation, dispersal, and so on. The complexity of the underlying genetic architecture per se may also contribute to the maintenance of genetic variation in the traits that underlie emergence pattern (Houle, 1991). However, to some extent the response to selection in such an experiment is likely to be confounded by within-host population dynamics, particularly if the 'fast' and 'slow' IJs that are selected are the product of different generations. For example, if all the IJs that emerged from a given host were the product of a single generation, selection for a 'slow' emerging line may favour parents that reproduce more slowly than a 'fast' line as well as traits in $\mathrm{IJ}$ s that lead to slower dispersal. But if several generations occur within the host, selecting for 'slow' and 'fast' lines may also select for developmental traits that are more particular to one generation than another - such as 
slower growth and resource assimilation in noninfective $F_{1}$ nematodes.

Stuart et al. (1996) suggested that 2-3 generations are likely to be produced within the host before IJs begin emerging in $S$. glaseri. Although the present study was conducted using a member of the Heterorhabditidae, our results stress the need to examine emergence patterns in the context of withinhost development. Moreover, Wang \& Bedding (1996) demonstrated that IJ development followed a similar temporal sequence in $H$. bacteriophora and $S$. carpocapsae. Similarly structured emergence patterns may thus occur in at least some members of the two families.

In conclusion, our results have elucidated several important potential in vivo determinants of variation in transmission success in an entomopathogenic nematode. Infection density exerts a strong constraint on the rate of offspring production across generations. However, the $P_{0}$ hermaphrodites appear able to offset this effect by investing preferentially in IJs at higher infection densities, when lower resource availability threatens to curtail the reproduction of non-infective offspring. Some of the consequences of this pattern of investment for the IJs that are produced form the subject of a separate study (Ryder \& Griffin, unpublished observations).

This work was supported by the Higher Education Authority, Ireland, under its Programme for Research in Third Level Institutions. Two anonymous referees provided comments that improved the manuscript.

\section{REFERENCES}

BOFF, M. I. C., WIEgERS, G. L., GERRITSEN, L. J. M. \& SMITS, P. H. (2000). Development of the entomopathogenic nematode Heterorhabditis megidis strain NLH-E 87.3 in Galleria mellonella. Nematology 2, 303-308.

Dix, I., KOlTAi, H., GLAZER, I. \& BURNELl, A. (1994). Sperm competition in mated first generation hermaphrodite females of the HP 88 strain of Heterorhabditis (Nematoda: Heterorhabditidae) and progeny sex ratios in mated and unmated females. Fundamental and Applied Nematology 17, 17-27. FODOR, A., VECSERI, G. \& FARKAS, T. (1990). Caenorhabditis elegans as a model for the study of entomopathogenic nematodes. In Entomopathogenic Nematodes in Biological Control (ed. Gaugler, R. \& Kaya, H. K.), pp. 249-265. CRC Press, Boca Raton, USA.

HOUlE, D. (1991). Genetic covariance of fitness correlates: what genetic correlations are made of and why it matters. Evolution 45, 630-648.

JARosz, J. (1998). Active resistance of entomophagous rhabditid Heterorhabditis bacteriophora to insect immunity. Parasitology 117, 201-208.
JOHNIGK, S.-A. \& EHLERS, R.-U. (1999a). Juvenile development and life cycle of Heterorhabditis bacteriophora and $H$. indica (Nematoda: Heterorhabditidae). Nematology 1, 251-260.

JOHNigK, S.-A. \& EHLERS, R.-U. (1999 b). Endotokia matricida in hermaphrodites of Heterorhabditis spp. and the effect of the food supply. Nematology $\mathbf{1}$, $717-726$.

KAHEL-RAIFER, H. \& GLAZER, I. (2000). Environmental factors affecting sexual differentiation in the entomopathogenic nematode Heterorhabditis bacteriophora. Fournal of Experimental Zoology 287, 158-166.

KOPPENHÖFER, A. M. \& KAYA, H. K. (1995). Densitydependent effects on Steinernema glaseri (Nematoda: Steinernematidae) within an insect host. Fournal of Parasitology 80, 797-799.

POINAR, G. O. Jr. (1990). Taxonomy and biology of Steinernematidae and Heterorhabditidae. In Entomopathogenic Nematodes in Biological Control (ed. Gaugler, R. \& Kaya, H. K.), pp. 23-61. CRC Press, Boca Raton, USA. ROFF, D. A. (1997). Evolutionary Quantitative Genetics, 1st Edn. Chapman and Hall, New York, USA. SElvan, s., CAMPBEll, J. F. \& GAUGler, R. (1993). Density-dependent effects on entomopathogenic nematodes (Heterorhabditidae and Steinernematidae) within an insect host. Fournal of Invertebrate Pathology 62, 278-284.

strauch, O., stoessel, s. \& Ehlers, R.-U. (1994). Culture conditions define automictic or amphimictic reproduction in entomopathogenic rhabditid nematodes of the genus Heterorhabditis. Fundamental and Applied Nematology 17, 575-582.

Stuart, R. J., LEWIS, E. E. \& GAUgler, R. (1996). Selection alters the pattern of emergence from the host cadaver in the entomopathogenic nematode, Steinernema glaseri. Parasitology 113, 183-189.

WANG, J. \& BEDding, R. A. (1996). Population development of Heterorhabditis bacteriophora and Steinernema carpocapsae in the larvae of Galleria mellonella. Fundamental and Applied Nematology 19, 363-367.

WOODRING, J. L. \& KAYA, H. K. (1988). Steinernematid and Heterorhabditid Nematodes: A Handbook of Biology and Techniques. Southern Cooperative Series Bulletin 331, Arkansas Experimental Station, Fayetteville, AR, USA.

UNDERWood, A. J. (1998). Experiments in Ecology, 1st Edn. Cambridge University Press, Cambridge, England.

ZERvos, s., JOHnson, s. C. \& WEBSTER, J. M. (1991). Effects of temperature and inoculum size on reproduction and development Heterorhabditis heliothidis and Steinernema glaseri (Nematoda: Rhabditoidea) in Galleria mellonella. Canadian Fournal of Zoology 69, 1261-1264. 